\title{
EGFR-mutated pulmonary adenocarcinoma with concurrent PIK3CA mutation, and with acquired RET fusion and EGFR T790M mutation after afatinib therapy
}

\author{
Minhye $\mathrm{Kim}^{1}$, Ji Min Na ${ }^{1}$, Gyeong-Won Lee ${ }^{2}$, Seung Jun Lee ${ }^{3}$, Jong Duk Kim4 ${ }^{4}$ Jung Wook Yang ${ }^{5}$ \\ 'Department of Pathology, Gyeonsang National University Hospital, Jinju; \\ ${ }^{2}$ Division of Hematology-Oncology, Department of Internal Medicine, \\ ${ }^{3}$ Division of Pulmonology and Allergy, Department of Internal Medicine, Gyeonsang National University Hospital, Gyeongsang National University College of Medicine, Jinju; \\ ${ }^{4}$ Department of Cardiothoracic Surgery, Gyeonsang National University Hospital, Jinju; \\ ${ }^{5}$ Department of Pathology, Gyeonsang National University Hospital, Gyeongsang National University College of Medicine, Jinju, Korea
}

Generally, receptor tyrosine kinase (RTK) fusions are mutually exclusive of epidermal growth factor receptor (EGFR) mutations in non-small cell lung cancer (NSCLC). However, RTK fusions have recently emerged as mechanisms of actionable resistance to EGFR-tyrosine kinase inhibitors (TKIs) in EGFR-mutated NSCLC [1]. More than half of the acquired RET fusions following EGFR-TKI therapy occur in response to osimertinib (third-generation) treatment. To the best of our knowledge, only one case of NSCLC with acquired RET fusion following afatinib (second-generation) therapy has been reported in the English literature. Phosphatidylinositol-4,5-bisphosphate 3-kinase catalytic subunit alpha (PIK3CA) mutations are found infrequently (approximately $7 \%$ ) in pulmonary adenocarcinoma [2]. Although other oncogenic driver mutations in NSCLC are mutually exclusive, PIK3CA mutations frequently coexist with other mutations [2,3]. Here, we report a rare case of $E G F R$-mutated pulmonary adenocarcinoma with concurrent PIK3CA mutation, displaying acquired RET fusion and EGFR T790M mutation following afatinib therapy.

\section{CASE REPORT}

A 64-year-old man without a known underlying disease was

Received: September 1, 2020 Revised: October 21, 2020

Accepted: November 2, 2020

Corresponding Author: Jung Wook Yang, MD, PhD

Department of Pathology, Gyeongsang National University Hospital, Gyeongsang

National University College of Medicine, 79 Gangnam-ro, Jinju 52727, Korea

Tel: +82-55-772-8065, Fax: +82-55-759-7952, E-mail: woogi1982@gmail.com transferred with dyspnea and pleural effusion. He was a smoker with a 30 pack-year history. The clinical course is shown in Fig. 1. The cytological examination of the pleural fluid confirmed the diagnosis as metastatic adenocarcinoma of the lung (Fig. 1A). Chest computed tomography (CT) (Fig. 2A) suggested lung malignancy with pleural and lymph node metastases. Positron emission tomography showed a 3-cm-sized hypermetabolic mass in the right upper lobe of the lung. CT-guided needle biopsy was performed on the mass in the right upper lobe, and adenocarcinoma was diagnosed (Fig. 1B). The EGFR mutation status was tested using real-time polymerase chain reaction clamping method on the pleural fluid and our results revealed exon 19 deletion. The patient received afatinib treatment, and as a result, the size of the lung mass and amount of pleural fluid were found to be decreased on chest CT after 3 months (Fig. 2B). However, the disease was stable for 6 months, and next-generation sequencing (NGS) was performed on the previous needle biopsy specimen of the lung on an Ion Torrent S5 sequencer (Thermo Fisher Scientific, Waltham, MA, USA) using a commercially available targeted gene panel (Oncomine Comprehensive Assay v3, Thermo Fisher Scientific). The NGS results showed exon 19 deletion of EGFR and PIK3CA G118D mutation. The disease progressed in 7.5 months (Fig. 2C) and afatinib had to be discontinued. Video-assisted thoracoscopic wedge resection was performed on the right upper lobe of the lung, and the status of the EGFR mutation was re-evaluated. Microscopic examination of the specimen showed tumor heterogeneity with solid and cribriform components (Fig. 1C), and acinar and papillary components (Fig. 1D). A second EGFR mutation test showed T790M 


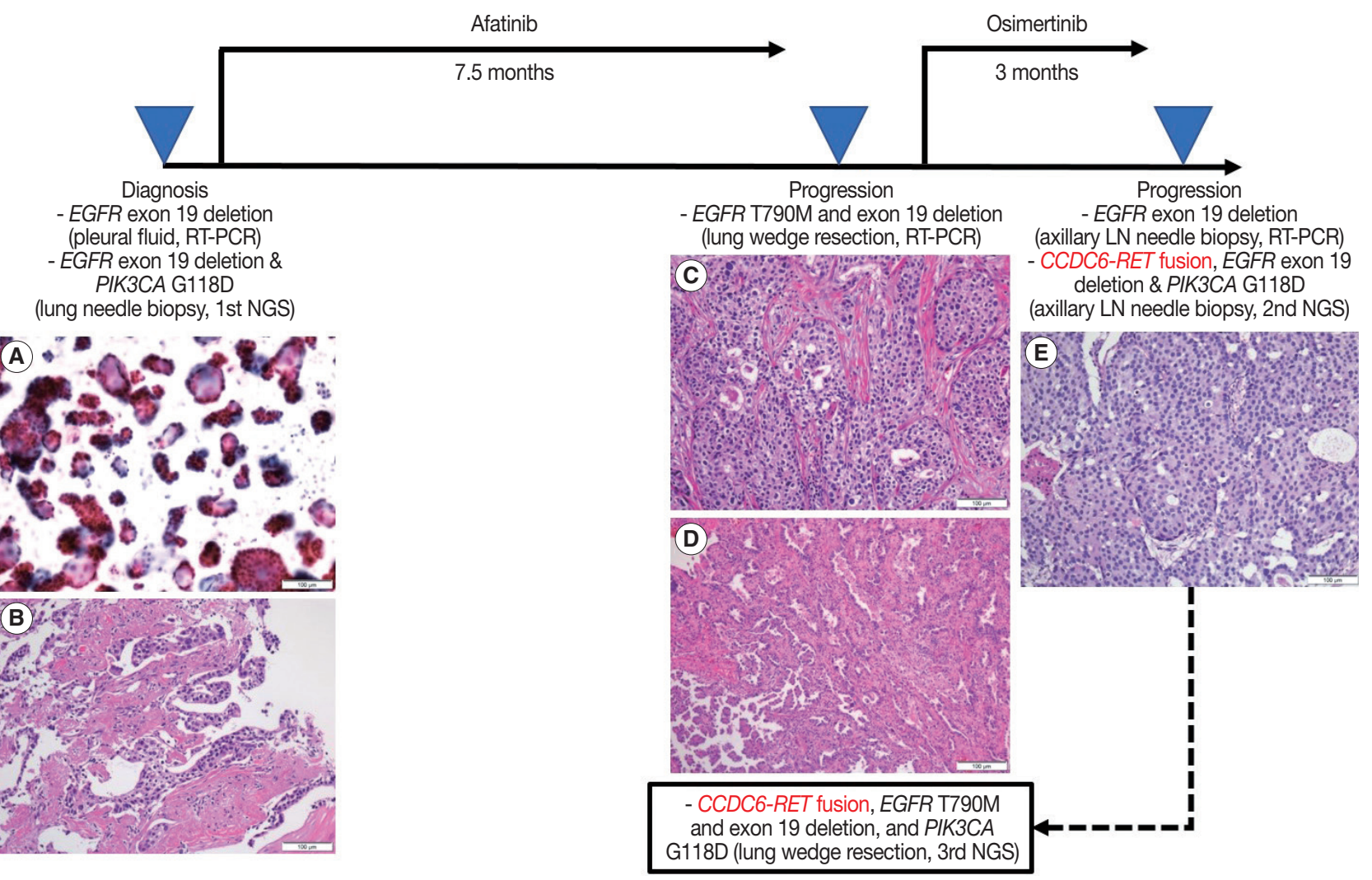

Fig. 1. Clinical course and pathological diagnosis of the patient. Adenocarcinoma was diagnosed using pleural fluid (A), and lung needle biopsy (B). Lung wedge resection specimen showed tumor heterogeneity; solid and cribriform components (C), and acinar and papillary components (D). (E) Metastatic adenocarcinoma was observed in the axillary LN. EGFR, epidermal growth factor receptor; PIK3CA, phosphatidylinositol-4,5-bisphosphate 3-kinase catalytic subunit alpha; NGS, next-generation sequencing; RT-PCR, reverse tranascription; LN, lymph node.

mutation and exon 19 deletion. The patient was prescribed with osimertinib, but the disease progressed in 3 months (Fig. 2D). Needle biopsy of the right axillary lymph node was performed for further molecular evaluation of the cancer. Metastatic adenocarcinoma with a solid and cribriform pattern was observed in the lymph node (Fig. 1E). The third EGFR mutation test showed exon 19 deletion but not T790M mutation. The second NGS test on axillary lymph node specimens revealed a new CCDC6-RET fusion in addition to exon 19 deletion of EGFR and PIK3CA G118D mutation. Additionally, a third NGS test was performed on the wedge resection specimen (second biopsy), which showed CCDC6-RET fusion with T790M mutation and exon 19 deletion of EGFR and PIK3CA G118D.

\section{DISCUSSION}

Viola et al. analyzed 86 cases of RTK fusions as acquired resistance in EGFR-mutated NSCLC [1]. The acquired RTK fusions occurred most frequently (57\%) after the third generation EGFR-TKI therapy (first, 24\%; second, 12\%). The most fre- quently reported acquired RTK fusion in EGFR-TKI-resistant NSCLC was RET fusion, whereas CCDC6-RET fusion was the most common variant. Combined EGFR and RET inhibition with osimertinib and BLU-667 may be an effective therapeutic strategy in EGFR-TKI-resistant NSCLC with acquired RTK fusion [4]. The concurrent PIK3CA mutation is a known poor prognostic and predictive marker for EGFR-TKI therapy in pulmonary adenocarcinomas [2]. CCDC6-RET fusion and PIK3CA G118D mutation are not the most common variants in NSCLC with a single oncogenic driver mutation $[1,5,6]$.

In this case, considering the third NGS result, the CCDC6RET fusion probably occurred with EGFR T790M mutation after afatinib treatment. In lung wedge specimens resected after afatinib treatment, we observed tumor heterogeneity (Fig. 1C, D). We could not confirm which tumor population had CCDC6RET fusion or EGFR T790M mutation or both. Considering morphological features (Fig. 1C, E), we assumed that tumor population with solid and cribriform patterns (Fig. 1C) had CCDC6RET fusion.

In conclusion, we report a rare case of EGFR-mutated pul- 

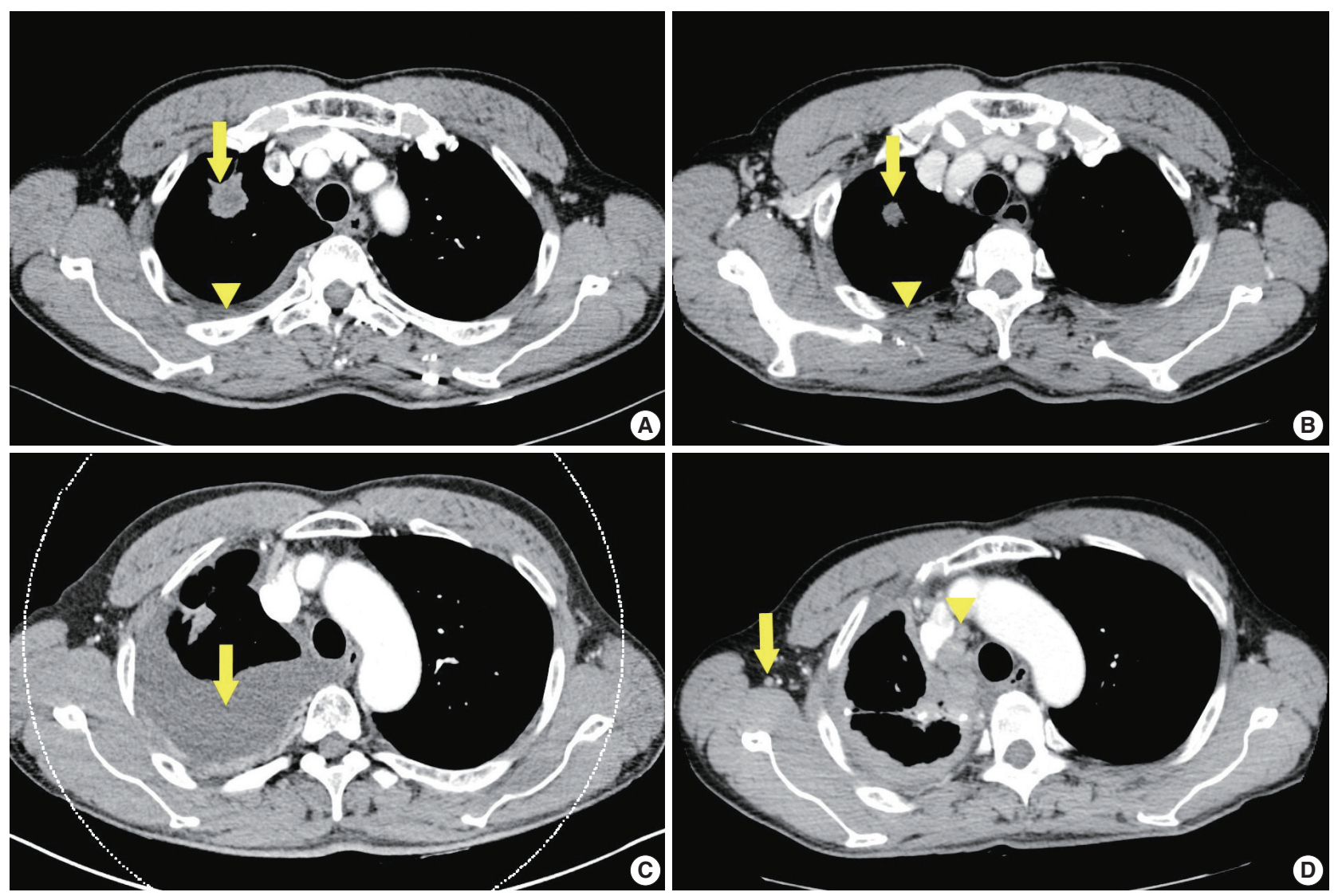

Fig. 2. Chest computed tomography. (A) Lung mass (arrow) in right upper lobe with pleural fluid (arrowhead) at diagnosis. (B) Decreased size of lung mass (arrow) and amount of pleural fluid (arrowhead) after 3 months of afatinib therapy. (C) Increased amount of pleural fluid (arrow) after 7.5 months with afatinib therapy. (D) Increased size of axillary (arrow) and mediastinal (arrowhead) lymph nodes after 3 months with osimertinib therapy.

monary adenocarcinoma with concurrent PIK3CA mutation, and acquired RET fusion and EGFR T790M mutation after EGFR-TKI therapy. Since NSCLC has a number of well-known oncogenic driver mutations, we believe that NGS is currently one of the best methods to determine the treatment of NSCLC, especially adenocarcinoma.

\section{Ethics Statement}

This study was approved by the Institutional Review Board of Gyeongsang National University Hospital, and informed consent was waived (IRB No. GNUH 2020-08-005).

\section{ORCID}

Minhye Kim https://orcid.org/0000-0002-8631-5104 Ji Min Na https://orcid.org/0000-0003-4330-6598 Gyeong-Won Lee https://orcid.org/0000-0002-1781-2262 Seung Jun Lee https://orcid.org/0000-0002-1849-5086 Jong Duk Kim https://orcid.org/0000-0003-0268-1674 Jung Wook Yang https://orcid.org/0000-0002-9698-3667

\section{Author Contributions}

Conceptualization: JWY. Data Curation: MK, JWY. Investigation: MK, JMN, JWY. Resources: GWL, SJL, JDK. Supervision: JWY. Visualization: MK, JWY. Writing —original draft preparation: MK, JWY. Writing-review and editing: MK, JWY. Approval of final manuscript: all authors.

\section{Conflicts of Interest}

The authors declare that they have no potential conflicts of interest.

\section{Funding Statement}

No funding to declare.

\section{References}

1. Zhu VW, Klempner SJ, Ou SI. Receptor tyrosine kinase fusions as an actionable resistance mechanism to EGFR TKIs in EGFR-mutant non-small-cell lung cancer. Trends Cancer 2019; 5: 677-92.

2. Eng J, Woo KM, Sima CS, et al. Impact of concurrent PIK3CA mutations on response to EGFR tyrosine kinase inhibition in EGFRmutant lung cancers and on prognosis in oncogene-driven lung adenocarcinomas. J Thorac Oncol 2015; 10: 1713-9.

3. Wang $\mathrm{L}, \mathrm{Hu} \mathrm{H}$, Pan Y, et al. PIK3CA mutations frequently coexist with EGFR/KRAS mutations in non-small cell lung cancer and sug- 
gest poor prognosis in EGFR/KRAS wildtype subgroup. PLoS One 2014; 9: e88291.

4. Piotrowska Z, Isozaki H, Lennerz JK, et al. Landscape of acquired resistance to osimertinib in EGFR-mutant NSCLC and clinical validation of combined EGFR and RET inhibition with osimertinib and BLU-667 for acquired RET fusion. Cancer Discov 2018; 8: 1529-39.

5. Scheffler M, Bos M, Gardizi M, et al. PIK3CA mutations in non- small cell lung cancer (NSCLC): genetic heterogeneity, prognostic impact and incidence of prior malignancies. Oncotarget 2015; 6: 1315-26.

6. Ahn JW, Kim HS, Yoon JK, et al. Identification of somatic mutations in EGFR/KRAS/ALK-negative lung adenocarcinoma in never-smokers. Genome Med 2014; 6: 18. 